\title{
Nonlinear $\mathscr{L}_{2}$-Gain Analysis of Hybrid Systems in the Presence of Sliding Modes and Impacts
}

\author{
T. Osuna, O. E. Montano, and Y. Orlov \\ Department of Electronics and Telecommunications, Center for Scientific Research and Higher Education at Ensenada, \\ Carretera Ensenada-Tijuana No. 3918, Zona Playitas, 22860 Ensenada, BC, Mexico
}

Correspondence should be addressed to Y. Orlov; yorlov@cicese.mx

Received 23 September 2015; Revised 3 December 2015; Accepted 17 December 2015

Academic Editor: Rui Wang

Copyright (C) 2016 T. Osuna et al. This is an open access article distributed under the Creative Commons Attribution License, which permits unrestricted use, distribution, and reproduction in any medium, provided the original work is properly cited.

The $\mathscr{L}_{2}$-gain analysis is extended towards hybrid mechanical systems, operating under unilateral constraints and admitting both sliding modes and collision phenomena. Sufficient conditions for such a system to be internally asymptotically stable and to possess $\mathscr{L}_{2}$-gain less than an a priori given disturbance attenuation level are derived in terms of two independent inequalities which are imposed on continuous-time dynamics and on discrete disturbance factor that occurs at the collision time instants. The former inequality may be viewed as the Hamilton-Jacobi inequality for discontinuous vector fields, and it is separately specified beyond and along sliding modes, which occur in the system between collisions. Thus interpreted, the former inequality should impose the desired integral input-to-state stability (iISS) property on the Filippov dynamics between collisions whereas the latter inequality is invoked to ensure that the impact dynamics (when the state trajectory hits the unilateral constraint) are input-to-state stable (ISS). These inequalities, being coupled together, form the constructive procedure, effectiveness of which is supported by the numerical study made for an impacting double integrator, driven by a sliding mode controller. Desired disturbance attenuation level is shown to satisfactorily be achieved under external disturbances during the collision-free phase and in the presence of uncertainties in the transition phase.

\section{Introduction}

Significant research interest has been devoted to the stability analysis and control synthesis of switched systems subject to input, state, and output constraints. The progress made in the area relied on different tools such as multiple Lyapunov functions [1] and predictive control [2]. More recently, barrier Lyapunov functions (functions which grow to infinity when their arguments approach the domain boundaries) have been involved in the tracking control synthesis of nonlinear switched systems with output constraints [3-6]. Sliding mode control of switched single-input, output-constrained systems has also been brought into play [7]. In addition, robustness of linear switched systems subject to actuator constraints has been studied in [8] in terms of $\mathscr{L}_{2}$-gain, using the LMI-optimization approach. A piecewise linear $\mathscr{H}_{\infty}$ control synthesis was developed for switched systems with output constraints in [9], relying again on the LMI-optimization.
Hybrid dynamic systems which are typically governed by a continuous differential equation and a difference equation, where the switch between such equations is defined according to output and/or time constraints, have also attracted a lot of attention due to the wide variety of their applications and due to the need of special analysis tools for this type of system. The interested reader may refer to the relevant works by Goebel et al. [10], Hamed and Grizzle [11], Naldi and Sanfelice [12], and Nešić et al. [13] to name a few. While admitting sliding modes and collision phenomena, hybrid dynamic systems possess nonsmooth solutions and a challenging problem is to extend popular robust technique such as the nonlinear $\mathscr{H}_{\infty}$ approach [14-16] to this kind of dynamic system.

It is worth noticing that potentially interesting Lyapunov characterizations of iISS of sliding modes [17] and that of impulsive systems [18] were confined to matched disturbances and to state-independent resets, respectively, thus suffering from the absence of the sliding mode analysis under 
both mismatched disturbances and state-dependent impacts. Recently, it was demonstrated by Castaños and Fridman [19] that the closed-loop system, driven in the sliding mode, is capable of presenting good performance in the presence of unmatched disturbances as well; however, $\mathscr{L}_{2}$-gain analysis of such systems has not been addressed yet. To avoid this shortcoming, $\mathscr{L}_{2}$-gain analysis was separately developed for sliding mode systems by Osuna and Orlov [20] and for dynamic systems under unilateral constraints by Montano et al. [21].

The present $\mathscr{L}_{2}$-gain analysis focuses on sliding mode mechanical systems, operating under unilateral constraints. Throughout, only unilateral constraints of codimension 1 are in play. The general case of multiunilateral constraints, possibly, resulting in ill-posed dynamics [22], calls for further investigation. $\mathscr{L}_{2}$-gain analysis, which has recently been developed by Orlov and Aguilar [23] towards nonsmooth mechanical applications with hard-to-model friction forces and backlash effects, is now extended in the presence of sliding modes and unilateral constraints.

Provided that the energy of the underlying mechanical system dissipates at the collision time instants when the unilateral constraint is attained, sufficient conditions for a hybrid system to be internally asymptotically stable and to possess $\mathscr{L}_{2}$-gain less than an a priori given disturbance attenuation level are carried out. These conditions are given in terms of appropriate solvability of a Hamilton-Jacobi partial differential inequality, separately viewed beyond and on the sliding manifold.

The proposed nonsmooth $\mathscr{L}_{2}$-gain analysis of hybrid mechanical systems in the presence of sliding modes and unilateral constraints constitutes the main contribution of the paper. An essential feature, adding the value to the present investigation, is that not only matched external disturbances (affecting the collision-free motion phase) but also their mismatched and discrete-time counterparts (which particularly occur in the collision phase) are attenuated with the proposed $\mathscr{L}_{2}$-gain test. In addition, this robustness feature is numerically justified in a benchmark double integrator, driven by a first-order sliding mode controller and impacting against a barrier.

The rest of the paper is outlined as follows. Section 2 presents a hybrid model of interest and its $\mathscr{L}_{2}$-gain analysis is developed. Capabilities of the proposed analysis are numerically illustrated in Section 3 for an impacting double integrator, driven by a sliding mode controller. Finally, conclusions and potential extensions of this work are presented in Section 4.

1.1. Notation and Preliminaries. The argument $t^{+}$is used to denote the right-hand side value $\mathbf{x}\left(t^{+}\right)$of a trajectory $\mathbf{x}(t)$ at an impact time instant $t$ whereas $\mathbf{x}\left(t^{-}\right)$stands for the left-hand side value of the same; by default, $\mathbf{x}(t)$ is reserved for $\mathbf{x}\left(t^{-}\right)$, thus implying an underlying trajectory to be continuous on the left. A standard notation

$$
\operatorname{DV}(\mathbf{x} ; \mathbf{l})=\lim _{\tau \rightarrow 0} \frac{V(\mathbf{x}+\tau \mathbf{l})-V(\mathbf{x})}{\tau}
$$

is for a Dini derivative of a scalar function $V(\mathbf{x})$, computed in the direction $\mathbf{l} \in \mathbb{R}^{n}$ at $\mathbf{x} \in \mathbb{R}^{n}$.

The following notion is due to Clarke [24]. A vector $\zeta(\widehat{\mathbf{x}}) \in$ $\mathbb{R}^{n}$ is a supergradient of a scalar function $\mathbf{f}(\mathbf{x})$ at $\widehat{\mathbf{x}} \in \mathbb{R}^{n}$ if there exists some $\boldsymbol{\sigma}(\widehat{\mathbf{x}})>\mathbf{0}$ such that

$$
\mathbf{f}(\mathbf{x}) \leq \mathbf{f}(\widehat{\mathbf{x}})+\zeta^{\top}(\widehat{\mathbf{x}})(\mathbf{x}-\widehat{\mathbf{x}})+\boldsymbol{\sigma}(\widehat{\mathbf{x}})\|\mathbf{x}-\widehat{\mathbf{x}}\|^{2}
$$

for all $\mathbf{x}$ in some neighborhood $U(\widehat{\mathbf{x}})$ of $\widehat{\mathbf{x}}$.

The set of supergradients at $\mathbf{x}$ is denoted by $\partial \mathbf{f}(\mathbf{x})$ and is referred to as the superdifferential.

For later use, a technical lemma is extracted from [25, Chapter 3].

Lemma 1. Let $\mathbf{x} \in \mathbb{R}^{n}$ be an absolutely continuous function of time variable $t$ and let $V(\mathbf{x})$ be a scalar locally Lipschitz function around $\mathbf{x} \in \mathbb{R}^{n}$. Then, the composite function $V(\mathbf{x})$ is absolutely continuous and its time derivative is given by

$$
\frac{\mathrm{d}}{\mathrm{d} t} V(\mathbf{x}(t))=\mathrm{DV}(\mathbf{x}(t), \dot{\mathbf{x}}(t))
$$

almost everywhere. Furthermore,

$$
\mathrm{DV}(\mathbf{x}(t), \dot{\mathbf{x}}(t)) \leq \frac{\partial V}{\partial \mathbf{x}} \dot{\mathbf{x}}(t)
$$

for almost all t and for all supergradients $(\partial V / \partial \mathbf{x})^{T} \in \partial V(\mathbf{x})$, if any.

\section{The Generic $\mathscr{L}_{2}$-Gain Analysis}

Given a scalar unilateral constraint $F\left(\mathbf{x}_{1}\right) \geq 0$, consider a nonlinear system, evolving within the above constraint, which is governed by continuous dynamics of the form

$$
\begin{aligned}
\dot{\mathbf{x}}_{1} & =\mathbf{x}_{2}+\psi_{1}\left(\mathbf{x}_{1}, \mathbf{x}_{2}\right) \mathbf{w}, \\
\dot{\mathbf{x}}_{2} & =\boldsymbol{\varphi}\left(\mathbf{x}_{1}, \mathbf{x}_{2}\right)+\psi_{2}\left(\mathbf{x}_{1}, \mathbf{x}_{2}\right) \mathbf{w}, \\
\mathbf{z} & =\mathbf{h}\left(\mathbf{x}_{1}, \mathbf{x}_{2}\right)
\end{aligned}
$$

beyond the surface $F\left(\mathbf{x}_{1}\right)=0$ when the constraint is inactive and by the algebraic relations

$$
\begin{aligned}
\mathbf{x}_{1}\left(t_{i}^{+}\right) & =\mathbf{x}_{1}\left(t_{i}^{-}\right), \\
\mathbf{x}_{2}\left(t_{i}^{+}\right) & =\boldsymbol{\mu}_{0}\left(\mathbf{x}_{1}\left(t_{i}\right), \mathbf{x}_{2}\left(t_{i}^{-}\right)\right)+\boldsymbol{\omega}\left(\mathbf{x}_{1}\left(t_{i}\right), \mathbf{x}_{2}\left(t_{i}^{-}\right)\right) \mathbf{w}_{\mathbf{i}}^{\mathbf{d}}, \\
\mathbf{z}_{\mathbf{i}}^{\mathbf{d}} & =\mathbf{x}_{2}\left(t_{i}^{+}\right)
\end{aligned}
$$

at $a$ priori unknown collision time instants $t=t_{i}, i=1,2, \ldots$, when the system trajectory hits the surface $F\left(\mathbf{x}_{1}\right)=0$.

In the above relations, $\mathbf{x}^{\top}=\left[\mathbf{x}_{1}^{\top}, \mathbf{x}_{2}^{\top}\right] \in \mathbb{R}^{2 n}$ represents the state vector with components $\mathbf{x}_{1} \in \mathbb{R}^{n}$ and $\mathbf{x}_{2} \in \mathbb{R}^{n} ; \mathbf{w} \in \mathbb{R}^{l}$ and $\mathbf{w}_{\mathbf{i}}^{\mathbf{d}} \in \mathbb{R}^{q}$ collect exogenous signals affecting the motion of the system (external forces, including impulsive ones, as well as model imperfections); the output variables $\mathbf{z} \in \mathbb{R}^{m}$ and $\mathbf{z}_{\mathbf{i}}^{\mathbf{d}} \in \mathbb{R}^{n}$ are responsible for the performance of the system.

Clearly, system (5)-(8) governs a wide class of mechanical systems with impacts and it is an affine system of the $l$-vector 
relative degree $[2, \ldots, 2]^{\top}$ with respect to the disturbance vector $w$ provided that $\psi_{1} \equiv 0$ and $\psi_{2} \neq 0$; for the sake of generality, $\psi_{1}$ is admitted to take nonzero values. If interpreted in terms of mechanical systems, (5) describes the continuous dynamics before the underlying system hits the reset surface $F\left(\mathbf{x}_{\mathbf{1}}\right)=0$, depending on the position only, whilst the restitution law, given by (7), is a physical law for the instantaneous change of the velocity when the resetting surface is hit. Thus, the position is not instantaneously changed at the collision time instants whereas the postimpact velocity $\mathbf{x}_{2}\left(t^{+}\right)$is a function of both the preimpact state $\left(\mathbf{x}_{1}(t), \mathbf{x}_{2}\left(t^{-}\right)\right)$and a discrete perturbation $\mathbf{w}_{\mathbf{d}}$ accounting for inadequacies of the restitution law.

Throughout, the matrix functions $\psi_{1}, \psi_{2}, \mathbf{h}, F, \boldsymbol{\mu}_{0}$, and $\boldsymbol{\omega}$ are of appropriate dimensions, which are continuously differentiable in their arguments, whereas the vector-function $\varphi \in$ $\mathbb{R}^{n}$ is piecewise continuously differentiable only. In addition, the origin is assumed to be an equilibrium of the unforced system (5)-(8), and $\mathbf{h}(\mathbf{0}, \mathbf{0})=\mathbf{0}$, and $\boldsymbol{\mu}_{0}(\mathbf{0}, \mathbf{0})=\mathbf{0}$.

For convenience of the reader, recall that the function $\varphi(\mathbf{x}): \mathbb{R}^{n} \mapsto \mathbb{R}^{n}$ is piecewise (locally Lipschitz) continuous if and only if $\mathbb{R}^{n}$ is partitioned into a finite number of domains $G_{j} \subset \mathbb{R}^{n}, j=1, \ldots, N$, with disjoint interiors and boundaries $\partial G_{j}$ of measure zero such that $\boldsymbol{\varphi}(\mathbf{x})$ is (locally Lipschitz) continuous within each of these domains and for all $j=$ $1, \ldots, N$ it has a finite limit $\varphi^{j}(\mathbf{x})$ as the argument $\mathbf{x}^{*} \in G_{j}$ approaches a boundary point $\mathbf{x} \in \partial G_{j}$.

As a matter of fact, the continuous dynamics (5) can be rewritten in the form

$$
\dot{\mathbf{x}}=\mathbf{f}(\mathbf{x})+\mathbf{g}(\mathbf{x}) \mathbf{w},
$$

whereas the restitution rule admits the representation

$$
\mathbf{x}\left(t_{i}^{+}\right)=\boldsymbol{\mu}\left(\mathbf{x}\left(t_{i}^{-}\right)\right)+\boldsymbol{\Omega}\left(\mathbf{x}\left(t_{i}^{-}\right)\right) \mathbf{w}_{\mathbf{i}}^{\mathbf{d}}, \quad i=1,2, \ldots,
$$

with $\mathbf{x}^{\top}=\left[\mathbf{x}_{1}^{\top}, \mathbf{x}_{2}^{\top}\right], \mathbf{f}^{\top}(\mathbf{x})=\left[\mathbf{x}_{2}^{\top}, \boldsymbol{\varphi}^{\top}(\mathbf{x})\right], \mathbf{g}^{\top}(\mathbf{x})=\left[\boldsymbol{\psi}_{1}^{\top}(\mathbf{x})\right.$, $\left.\psi_{2}^{\top}(\mathbf{x})\right], \boldsymbol{\mu}^{\top}(\mathbf{x})=\left[\mathbf{x}_{1}^{\top}, \boldsymbol{\mu}_{0}^{\top}(\mathbf{x})\right]$, and $\boldsymbol{\Omega}^{\top}(\mathbf{x})=[\mathbf{0}, \boldsymbol{\omega}(\mathbf{x})]$.

The precise meaning of the differential equation

$$
\dot{\mathbf{x}}=\mathbf{f}(\mathbf{x}),
$$

with a piecewise continuous right-hand side, is throughout defined in the sense of Filippov. For convenience of the reader, the following definition is recalled from Filippov [26].

Definition 2. Given the differential equation (11), let one introduce for each point $\mathbf{x} \in \mathbb{R}^{n}$ the smallest convex closed set $\mathscr{F}(\mathbf{x})$ which contains all the limit points of $\mathbf{f}\left(\mathbf{x}^{*}\right)$ as $\mathbf{x}^{*} \rightarrow \mathbf{x}$, and $\mathbf{x}^{*} \in \mathbb{R}^{n} \backslash\left(\cup_{j=1}^{N} \partial G_{j}\right)$. An absolutely continuous function $\mathbf{x}$ is said to be a solution of (11) if it satisfies the differential inclusion

$$
\dot{\mathbf{x}} \in \mathscr{F}(\mathbf{x}) .
$$

Note that the extension of the Filippov solution concept to the perturbed differential equation (9) is straightforward.

At any continuity point $\mathbf{x} \in \cup_{i=1}^{N} G_{i}$ of the function $\mathbf{f}$ the Filippov set $\mathscr{F}(\mathbf{x})$ consists of the only point $\mathbf{f}(\mathbf{x})$, and the Filippov solution satisfies (11) in the conventional sense.
If the function $\mathbf{f}(\mathbf{x})$ undergoes discontinuities on a smooth surface $S$, governed by a scalar equation $s(\mathbf{x})=0$, then the discontinuity set $S$ separates $\mathbf{x}$ space into domains $G^{-}=\{\mathbf{x} \epsilon$ $\left.\mathbb{R}^{n}: s(\mathbf{x})<0\right\}$ and $G^{+}=\left\{\mathbf{x} \in \mathbb{R}^{n}: s(\mathbf{x})>0\right\}$, and the Filippov set $\boldsymbol{\Phi}(\mathbf{x})$ is a linear segment joining the endpoints of the vectors

$$
\begin{aligned}
\mathbf{f}^{-}(\mathbf{x}) & =\lim _{\xi \in G^{-}, \xi \rightarrow \mathbf{x}} \mathbf{f}(\boldsymbol{\xi}), \\
\mathbf{f}^{+}(\mathbf{x}) & =\lim _{\xi \in G^{+}, \xi \rightarrow \mathbf{x}} \mathbf{f}(\boldsymbol{\xi}) .
\end{aligned}
$$

According to Definition 2, a sliding mode on the discontinuity set $s(\mathbf{x})=0$, if any, is governed by

$$
\dot{\mathbf{x}}=\mathbf{f}^{0}(\mathbf{x})+\mathbf{g}^{0}(\mathbf{x}) \mathbf{w},
$$

where the intersection of the Filippov segment $\mathscr{F}(\mathbf{x})$ and the plane $T$, tangential to $S$, determines the endpoint of the vector $\mathbf{f}^{\mathbf{0}}(\mathbf{x})$. Analytically, this vector is expressed in the form

$$
\begin{aligned}
\mathbf{f}^{\mathbf{0}}(\mathbf{x})+\mathbf{g}^{\mathbf{0}}(\mathbf{x}) \mathbf{w}= & \boldsymbol{\kappa}(\mathbf{x}) \mathbf{f}^{+}(\mathbf{x})+[\mathbf{1}-\boldsymbol{\kappa}(\mathbf{x})] \mathbf{f}^{-}(\mathbf{x}) \\
& +\mathbf{g}(\mathbf{x}) \mathbf{w},
\end{aligned}
$$

where

$$
\boldsymbol{\kappa}(\mathbf{x})=\frac{\operatorname{grad}^{\top} s(\mathbf{x})\left[\mathbf{f}^{-}(\mathbf{x})+\mathbf{g}(\mathbf{x}) \mathbf{w}\right]}{\operatorname{grad}^{\top} s(\mathbf{x})\left[\mathbf{f}^{-}(\mathbf{x})-\mathbf{f}^{+}(\mathbf{x})\right]}
$$

is found from the condition

$$
\begin{aligned}
& \operatorname{grad}^{\top} s(\mathbf{x})\left\{\boldsymbol{\kappa}(\mathbf{x}) \mathbf{f}^{+}(\mathbf{x})+[\mathbf{1}-\boldsymbol{\kappa}(\mathbf{x})] \mathbf{f}^{-}(\mathbf{x})+\mathbf{g}(\mathbf{x}) \mathbf{w}\right\} \\
& \quad=0
\end{aligned}
$$

where the velocity vector (15) is in the plane $T$, tangential to $S$. To summarize, the relations

$$
\begin{aligned}
\mathbf{f}^{\mathbf{0}}(\mathbf{x})= & \frac{\operatorname{grad}^{\top} s(\mathbf{x}) \mathbf{f}^{-}(\mathbf{x})}{\operatorname{grad}^{\top} s(\mathbf{x})\left[\mathbf{f}^{-}(\mathbf{x})-\mathbf{f}^{+}(\mathbf{x})\right]} \mathbf{f}^{+}(\mathbf{x}) \\
& -\frac{\operatorname{grad}^{\top} s(\mathbf{x}) \mathbf{f}^{+}(\mathbf{x})}{\operatorname{grad}^{\top} s(\mathbf{x})\left[\mathbf{f}^{-}(\mathbf{x})-\mathbf{f}^{+}(\mathbf{x})\right]} \mathbf{f}^{-}(\mathbf{x}), \\
\mathbf{g}^{\mathbf{0}}(\mathbf{x}) \mathbf{w}= & \mathbf{g}(\mathbf{x}) \mathbf{w}+\frac{\operatorname{grad}^{\top} s(\mathbf{x}) \mathbf{g}(\mathbf{x}) \mathbf{w}}{\operatorname{grad}^{\top} s(\mathbf{x})\left[\mathbf{f}^{-}(\mathbf{x})-\mathbf{f}^{+}(\mathbf{x})\right]} \mathbf{f}^{+}(\mathbf{x}) \\
& +\left[1-\frac{\operatorname{grad}^{\top} s(\mathbf{x}) \mathbf{g}(\mathbf{x}) \mathbf{w}}{\operatorname{grad}^{\top} s(\mathbf{x})\left[\mathbf{f}^{-}(\mathbf{x})-\mathbf{f}^{+}(\mathbf{x})\right]}\right] \mathbf{f}^{-}(\mathbf{x})
\end{aligned}
$$

are derived to determine the functions $\mathbf{f}^{\mathbf{0}}(\mathbf{x})$ and $\mathbf{g}^{\mathbf{0}}(\mathbf{x})$.

Our objective is to develop $\mathscr{L}_{2}$-gain analysis of the hybrid system (9) with the restitution rule (10) on the unilateral constraint surface $F\left(\mathbf{x}_{1}\right)=0$ and with the performance output, specified by (6), (8). The analysis to be developed is made under the following assumptions, imposed on the underlying system:

(A1) The functions $\mathbf{g}^{\top}(\mathbf{x})$ and $\mathbf{h}(\mathbf{x})$ are locally Lipschitz continuous whereas $\mathbf{g}(\mathbf{x})$ is piecewise locally Lipschitz continuous. 
(A2) The origin is assumed to be an equilibrium of the unforced system (11), and $\mathbf{h}(\mathbf{0})=\mathbf{0}$, and $\boldsymbol{\mu}(\mathbf{0})=\mathbf{0}$.

(A3) The function $\mathbf{f}(\mathbf{x})$ undergoes discontinuities on a smooth surface $S$, governed by the scalar equation

$$
s(\mathbf{x})=0 .
$$

We are now in position to introduce $\mathscr{L}_{2}$-gain concept for the underlying discontinuous system (5)-(8), equivalently represented in the generic form (9), (10).

Definition 3. Given a real number $\gamma>0$, referred to as a disturbance attenuation level, it is said that the generic system (9), (10) locally possesses $\mathscr{L}_{2}$-gain less than $\gamma$ with respect to outputs (6), (8) if the inequality

$$
\begin{aligned}
& \int_{0}^{T}\|\mathbf{z}(t)\|^{2} \mathrm{~d} t+\sum_{i=1}^{N_{T}}\left\|\mathbf{z}_{\mathbf{i}}^{\mathbf{d}}\right\|^{2} \\
& \leq \gamma^{2}\left[\int_{0}^{T}\|\mathbf{w}(t)\|^{2} \mathrm{~d} t+\sum_{i=1}^{N_{T}}\left\|\mathbf{w}_{\mathbf{i}}^{\mathbf{d}}\right\|^{2}\right]+\beta_{0}(\mathbf{x}(0)) \\
& +\sum_{k=1}^{N_{T}} \beta_{k}\left(\mathbf{x}\left(t_{k}^{-}\right)\right)
\end{aligned}
$$

holds for some positive definite functions $\beta_{k}(\mathbf{x}), k=$ $0, \ldots, N_{T}$, for all segments $T>0$ and natural $N_{T}$ such that $t_{N_{T}} \leq T<t_{N_{T}+1}$, for all piecewise continuous disturbances $\mathbf{w}(t)$ and discrete ones $\mathbf{w}_{\mathbf{i}}^{\mathbf{d}}, i=1,2, \ldots$, for which the state trajectory of the underlying system starting from an initial point $\mathbf{x}\left(t_{0}\right)=\mathbf{x}_{\mathbf{0}} \in \mathcal{U}$ remains in some neighborhood $\mathcal{U} \epsilon$ $\mathbb{R}^{2 n}$ of the origin for all $t \in[0, T]$.

It is worth noticing that the above $\mathscr{L}_{2}$-gain definition is consistent with the notion of dissipativity, introduced by Willems [27] and Hill and Moylan [28], and with iISS notion, Hespanha et al. [18], and it represents a natural extension to hybrid systems (see, e.g., the works by Nešić et al. [29], Yuliar et al. [30], Lin and Byrnes [31], and Baras and James [32]). In order to facilitate the exposition, the underlying system, chosen for treatment, has been prespecified with the postimpact velocity value $\mathbf{x}_{2}(t)$ in the discrete output (8). The general case of a certain function $\boldsymbol{\kappa}\left(\mathbf{x}_{2}(t)\right)$ of the postimpact velocity value in the discrete output (8) can be treated in a similar manner because $\mathscr{L}_{2}$-gain inequality (21) is flexible in the choice of positive definite functions $\beta_{k}(\mathbf{x}), k=0, \ldots, N_{T}$.

\subsection{Hamilton-Jacobi Inequality and Its Proximal Solutions.} The Hamilton-Jacobi inequality

$$
\begin{gathered}
\frac{\partial V}{\partial \mathbf{x}} \boldsymbol{\varphi}(\mathbf{x})+\frac{1}{4 \gamma^{2}} \frac{\partial V}{\partial \mathbf{x}} \boldsymbol{\psi}(\mathbf{x}) \psi^{\top}(\mathbf{x})\left(\frac{\partial V}{\partial \mathbf{x}}\right)^{\top} \\
+\mathbf{h}^{\top}(\mathbf{x}) \mathbf{h}(\mathbf{x}) \leq-v(\mathbf{x})
\end{gathered}
$$

with some positive $\gamma$ and some positive definite function $\nu(\mathbf{x})$ is introduced in a standard manner within the continuity regions $G^{-}$and $G^{+}$(i.e., outside the discontinuity surface $S$ ) whereas on the discontinuity surface (20), inequality (22) is specified according to (15) with

$$
\varphi(\mathbf{x})=\varphi^{0}(\mathbf{x}) \quad \text { provided that } s(\mathbf{x})=0 .
$$

In other words, the Hamilton-Jacobi inequality, if confined to the discontinuity surface (20), takes the form

$$
\begin{gathered}
\frac{\partial V}{\partial \mathbf{x}} \boldsymbol{\varphi}^{0}(\mathbf{x})+\frac{1}{4 \gamma^{2}} \frac{\partial V}{\partial \mathbf{x}} \psi(\mathbf{x}) \psi^{\top}(\mathbf{x})\left(\frac{\partial V}{\partial \mathbf{x}}\right)^{\top} \\
+\mathbf{h}^{\top}(\mathbf{x}) \mathbf{h}(\mathbf{x}) \leq-\nu(\mathbf{x}) .
\end{gathered}
$$

Definition 4. A locally Lipschitz continuous function $V(\mathbf{x})$ is said to be a (local) proximal solution of the partial differential inequality (22), specified on the discontinuity manifold (23) according to (15) if and only if its proximal superdifferential $\partial^{P} V(\mathbf{x})$ is everywhere nonempty and (22) holds with $V(\mathbf{x})$ beyond the discontinuity surface (20) (locally around the origin) for all proximal supergradients $\partial V / \partial \mathbf{x} \in \partial^{P} V(\mathbf{x})$ whereas the sliding mode Hamilton-Jacobi inequality (24) is satisfied on the discontinuity surface (20) (locally around the origin) for all $\partial V / \partial \mathbf{x} \in \partial^{P} V(\mathbf{x})$.

The interested reader may refer to Clarke [24] for the proximal superdifferential concept for continuous vector fields.

Let $B_{\delta}^{2 n} \in \mathbb{R}^{2 n}$ be a ball of radius $\delta>0$, centered around the origin. Given $\gamma>0, \mathscr{L}_{2}$-gain analysis of the hybrid system (9), (10) with respect to outputs (6), (8) is made under the hypotheses, specified below in a domain $\mathbf{x} \in B_{\delta}^{2 n}$ of interest:

(H1) The norm of the matrix function $\omega$ is upper bounded by $(\sqrt{2} / 2) \gamma$; that is,

$$
\|\boldsymbol{\omega}(\mathbf{x})\| \leq \frac{\sqrt{2}}{2} \gamma
$$

(H2) The Hamilton-Jacobi inequality, given by (22) beyond the discontinuity surface (20) and specified with (15) and (23) along this surface, possesses a local positive definite proximal (Lipschitz continuous!) solution $V(\mathbf{x})$ under some positive $\gamma$ and some positive definite function $\nu(\mathbf{x})$.

(H3) Hypothesis (H2) is satisfied with the function $V(\mathbf{x})$ which decreases along the direction $\boldsymbol{\mu}$ in the sense that the inequality

$$
V(\mathbf{x}, t) \geq V(\boldsymbol{\mu}(\mathbf{x}, t), t)
$$

holds in the domain of $V$.

The following result is in force.

Theorem 5. Consider the hybrid system (6), (8), (9), and (10) with Assumptions (A1)-(A3). Given $\gamma>0$, suppose that Hypotheses (H1) and (H2) are satisfied for the system in a domain $\mathbf{x} \in B_{\delta}^{2 n}$ with a function $V(\mathbf{x})$. Then, the hybrid system (9), (10) locally possesses $\mathscr{L}_{2}$-gain less than $\gamma$ with respect to outputs (6), (8). Once Hypothesis (H3) is satisfied as well, the asymptotic stability of the disturbance-free version of the hybrid system (9), (10) is additionally guaranteed. 
2.2. Proof of Theorem 5. The proof of Theorem 5 is preceded with an instrumental lemma which extends the powerful Lyapunov approach to impact systems. The following result specifies [33, Theorem 2.4] to the present case with $x_{1}=x$ and $x_{2}=t$.

Lemma 6. Consider the unforced $(\mathbf{u}=\mathbf{0})$ disturbance-free $\left(\mathbf{w}=\mathbf{0}, \mathbf{w}_{\mathbf{i}}^{\mathbf{d}}=\mathbf{0}, i=1,2, \ldots\right)$ system (9), (10) with the assumptions above. Let there exist a positive definite function $V(\mathbf{x})$ such that its time derivative, computed along (9), is negative definite whereas $V(\mathbf{x}) \geq V(\boldsymbol{\mu}(\mathbf{x}))$ for all $\mathbf{x} \in \mathbb{R}^{2 n}$. Then, the system is asymptotically stable.

Proof of Theorem 5. The proof is rather technical and it follows the standard arguments of the nonlinear $\mathscr{L}_{2}$-gain analysis of Isidori and Astolfi [15] and Van Der Schaft [16], recently extended in Osuna and Orlov [20] to discontinuous (Filippov) vector fields and Montano et al. [21] to dynamic systems, operating under unilateral constraints. It is clear that Lemma 1 is applicable both to a proximal solution $V(\mathbf{x})$ of the Hamilton-Jacobi inequality (22), viewed on the solutions $\mathbf{x}(t)$ of the disturbance-free system (11) beyond the discontinuity manifold (20), and to that of (24), viewed on the solutions of the disturbance-free system (14) when $\mathbf{g}^{\mathbf{0}}(\mathbf{x})=\mathbf{0}$ along the discontinuity manifold (20). Then, relations (3), (4), (22), and (24), coupled together, result in

$$
\frac{\mathrm{d}}{\mathrm{d} t} V(\mathbf{x})=\mathrm{DV}(\mathbf{x}, \dot{\mathbf{x}}) \leq \frac{\partial V}{\partial \mathbf{x}} \dot{\mathbf{x}}=\frac{\partial V}{\partial \mathbf{x}} \boldsymbol{\varphi}(\mathbf{x}) \leq-v(\mathbf{x}) .
$$

With (27) in mind, Hypotheses (H2) and (H3) ensure that Lemma 6 is applicable to the disturbance-free version of the hybrid system (9), (10), which is thus shown to be asymptotically stable.

It remains to demonstrate that the disturbed system (9), (10) locally possesses $\mathscr{L}_{2}$-gain less than $\gamma$ with respect to outputs (6), (8). For this purpose, let us first focus on the system dynamics beyond the discontinuity manifold (20) and let us introduce the multivalued function

$$
\begin{aligned}
H(\mathbf{x}, \mathbf{w})= & \frac{\partial V(\mathbf{x})}{\partial \mathbf{x}}[\mathbf{f}(\mathbf{x})+\mathbf{g}(\mathbf{x}) \mathbf{w}]+\mathbf{h}^{\top}(\mathbf{x}) \mathbf{h}(\mathbf{x}) \\
& -\gamma^{2} \mathbf{w}^{\top} \mathbf{w},
\end{aligned}
$$

where $\partial V / \partial \mathbf{x} \in \partial^{P} V(\mathbf{x})$. Clearly, the multivalued function (28) is quadratic in $\mathbf{w}$. Then,

$$
\left.\frac{\partial H(\mathbf{x}, \mathbf{w})}{\partial \mathbf{w}}\right|_{\mathbf{w}=\boldsymbol{\alpha}(\mathbf{x})}=\frac{\partial V(\mathbf{x})}{\partial \mathbf{x}} \mathbf{g}(\mathbf{x})-2 \gamma^{2} \boldsymbol{\alpha}^{\top}(\mathbf{x})=\mathbf{0}
$$

for $\partial V / \partial \mathbf{x} \in \partial^{P} V(\mathbf{x})$ and

$$
\boldsymbol{\alpha}^{\mathrm{T}}(\mathbf{x})=\frac{1}{2 \gamma^{2}} \frac{\partial V(\mathbf{x})}{\partial \mathbf{x}} \mathbf{g}(\mathbf{x})
$$

Expanding the quadratic function $H(\mathbf{x}, \mathbf{w})$ in Taylor series, we derive that

$$
H(\mathbf{x}, \mathbf{w})=H(\mathbf{x}, \boldsymbol{\alpha}(\mathbf{x}))-\gamma^{2}\|\mathbf{w}-\boldsymbol{\alpha}(\mathbf{x})\|^{2},
$$

where $H(\mathbf{x}, \boldsymbol{\alpha}(\mathbf{x})) \leq-v(\mathbf{x})$ due to (22). Hence,

$$
H(\mathbf{x}, \mathbf{w}) \leq-\gamma^{2}\|\mathbf{w}-\boldsymbol{\alpha}(\mathbf{x})\|^{2}-v(\mathbf{x})
$$

and employing (28) and (31) we arrive at

$$
\begin{aligned}
& \frac{\partial V(\mathbf{x})}{\partial \mathbf{x}}[\mathbf{f}(\mathbf{x})+\mathbf{g}(\mathbf{x}) \mathbf{w}] \\
& \quad \leq-\gamma^{2}\|\mathbf{w}-\boldsymbol{\alpha}(\mathbf{x})\|^{2}-\nu(\mathbf{x})-\|\mathbf{h}(\mathbf{x})\|^{2}+\gamma^{2}\|\mathbf{w}\|^{2} .
\end{aligned}
$$

By applying Lemma 1 and taking (33) into account, a time derivative estimate of the solution $V(\mathbf{x})$ of the HamiltonJacobi inequality (22) on the trajectories of (9) between the collision time instants is given as

$$
\frac{\mathrm{d}}{\mathrm{d} t} V(\mathbf{x}) \leq-\gamma^{2}\|\mathbf{w}-\boldsymbol{\alpha}(\mathbf{x})\|^{2}-\nu(\mathbf{x})-\|\mathbf{z}\|^{2}+\gamma^{2}\|\mathbf{w}\|^{2} .
$$

Following the same line of reasoning, estimate (34) is additionally verified for the system dynamics along the discontinuity manifold (20) with $\boldsymbol{\alpha}(\mathbf{x})$ subject to (30), now specified with $\mathbf{g}(\mathbf{x})=\mathbf{g}^{\mathbf{0}}(\mathbf{x})$, where $\mathbf{g}^{\mathbf{0}}(\mathbf{x})$ is given by (19).

By integrating (34) between the collision time instants $t_{k}$ and $t_{k+1}, k=0,1, \ldots$, it follows that

$$
\begin{aligned}
& \int_{t_{k}}^{t_{k+1}}\left[\gamma^{2}\|\mathbf{w}\|^{2}-\|\mathbf{z}(t)\|^{2}\right] \mathrm{d} t \\
& \geq \int_{t_{k}}^{t_{k+1}} \nu(\mathbf{x}(t)) \mathrm{d} t+\int_{t_{k}}^{t_{k+1}} \frac{\mathrm{d} V(\mathbf{x}(t))}{\mathrm{d} t} \mathrm{~d} t \\
& \quad+\gamma^{2} \int_{t_{k}}^{t_{k+1}}\|\mathbf{w}(t)-\boldsymbol{\alpha}(\mathbf{x}(t), t)\|^{2} \mathrm{~d} t>0 .
\end{aligned}
$$

Skipping positive terms in the right-hand side of (35) yields

$$
\begin{aligned}
& \int_{0}^{T}\left(\gamma^{2}\|\mathbf{w}\|^{2}-\|\mathbf{z}(t)\|^{2}\right) \mathrm{d} t \\
& \geq V(\mathbf{x}(T))+\sum_{i=1}^{N_{T}}\left[V\left(\mathbf{x}\left(t_{i}^{-}\right)\right)-V\left(\mathbf{x}\left(t_{i}^{+}\right)\right)\right] \\
& \quad-V\left(\mathbf{x}\left(t_{0}\right)\right) .
\end{aligned}
$$

Since the function $V$ is Lipschitz continuous by Hypothesis (H2), the relation

$$
\begin{aligned}
& \left|V\left(\mathbf{x}\left(t_{i}^{-}\right)\right)-V\left(\mathbf{x}\left(t_{i}^{+}\right)\right)\right| \leq L_{\delta}\left\|\mathbf{x}\left(t_{i}^{-}\right)-\mathbf{x}\left(t_{i}^{+}\right)\right\| \\
& \quad \leq L_{\delta}\left[\left\|\mathbf{x}\left(t_{i}^{-}\right)\right\|+\left\|\mathbf{x}\left(t_{i}^{+}\right)\right\|\right]
\end{aligned}
$$

holds true with $L_{\delta}>0$ being Lipschitz constant of $V$ in the domain $B_{\delta}^{2 n} \in \mathbb{R}^{2 n}$. Relations (36) and (37), coupled together, ensure the inequality

$$
\begin{aligned}
& \int_{t_{0}}^{T}\left(\gamma^{2}\|\mathbf{w}\|^{2}-\|\mathbf{z}(t)\|^{2}\right) \mathrm{d} t \\
& \quad \geq-2 L_{\delta} \sum_{i=1}^{N_{T}}\left\|\mathbf{x}\left(t_{i}^{-}\right)\right\|-V(\mathbf{x}(0))
\end{aligned}
$$


in the domain $B_{\delta}^{2 n} \in \mathbb{R}^{2 n}$. Apart from this, inequality

$$
\begin{aligned}
\sum_{i=1}^{N_{T}}\left\|\mathbf{z}_{\mathbf{i}}^{\mathbf{d}}\right\|^{2} & =\sum_{i=1}^{N_{T}}\left\|\mathbf{x}_{\mathbf{2}}\left(t_{i}^{+}\right)\right\|^{2} \\
& \leq 2 \sum_{i=1}^{N_{T}}\left\|\boldsymbol{\mu}_{\mathbf{0}}\left(\mathbf{x}\left(t_{i}^{-}\right)\right)\right\|^{2}+2 \sum_{i=1}^{N_{T}}\left\|\mathbf{w}\left(\mathbf{x}\left(t_{i}^{-}\right)\right) \mathbf{w}_{\mathbf{i}}^{\mathbf{d}}\right\|^{2} \\
& \leq 2 \sum_{i=1}^{N_{T}}\left\|\boldsymbol{\mu}_{\mathbf{0}}\left(\mathbf{x}\left(t_{i}^{-}\right)\right)\right\|^{2}+\gamma^{2} \sum_{i=1}^{N_{T}}\left\|\mathbf{w}_{\mathbf{i}}^{\mathbf{d}}\right\|^{2}
\end{aligned}
$$

is ensured by Hypothesis (H1). Thus, combining (38) and (39), one derives

$$
\begin{aligned}
\int_{t_{0}}^{T}\|\mathbf{z}(t)\|^{2} \mathrm{~d} t+\sum_{i=1}^{N_{T}}\left\|\mathbf{z}_{\mathbf{i}}^{\mathbf{d}}\right\|^{2} \\
\leq V(\mathbf{x}(0))+\gamma^{2}\left[\int_{t_{0}}^{T}\|\mathbf{w}(t)\|^{2} \mathrm{~d} t+\sum_{i=1}^{N_{T}}\left\|\mathbf{w}_{\mathbf{i}}^{\mathbf{d}}\right\|^{2}\right] \\
\quad+2 \sum_{i=1}^{N_{T}}\left\|\boldsymbol{\mu}_{\mathbf{0}}\left(\mathbf{x}\left(t_{i}^{-}\right)\right)\right\|^{2}+2 L_{\delta} \sum_{i=1}^{N_{T}}\left\|\mathbf{x}\left(t_{i}^{-}\right)\right\| ;
\end{aligned}
$$

that is, the disturbance attenuation inequality (21) is established with the positive definite functions

$$
\begin{aligned}
& \beta_{0}(\mathbf{x})=V(\mathbf{x}), \\
& \beta_{i}(\mathbf{x})=2 L_{\delta}\|\mathbf{x}\|+2\left\|\boldsymbol{\mu}_{\mathbf{0}}(\mathbf{x})\right\|^{2}, \\
& \quad i=1, \ldots, N .
\end{aligned}
$$

Theorem 5 is thus proved.

While proving Theorem 5, it is established that the proximal solution $V(x)$ of the Hamilton-Jacobi inequality represents a Lyapunov function of the undisturbed system. Once the plant equations are linear, a Lyapunov function can be sought in the quadratic form, and its specific expression is obtained by solving the corresponding Hamilton-Jacobi inequality (22). This is however not generally true, and for switched systems, absolute-value functions become useful in combination with quadratic ones. Such a combined Lyapunov function is further utilized to tune sliding mode controller gains while regulating a double integrator to the impact surface.

\section{A Case Study: Impacting Double Integrator Driven by Sliding Mode Controller}

3.1. Model of the Plant. To support our theoretical results, system (9), (10) is specified in the state space $x \in \mathbb{R}^{2}$ with

$$
\begin{aligned}
\mathbf{x} & =\left[\begin{array}{ll}
x_{1} & x_{2}
\end{array}\right]^{\top}, \\
\mathbf{w} & =\left[\begin{array}{ll}
w_{1} & w_{2}
\end{array}\right]^{\top}, \\
\boldsymbol{\mu}(\mathbf{x}) & =\left[\begin{array}{ll}
x_{1} & -e x_{2}
\end{array}\right]^{\top}, \\
\boldsymbol{\Omega}(\mathbf{x}) & =\left[\begin{array}{ll}
0 & \epsilon
\end{array}\right]^{\top},
\end{aligned}
$$

$$
\begin{aligned}
& \mathbf{f}(\mathbf{x})=\left[\begin{array}{ll}
x_{2} & -M \operatorname{sign}\left(c x_{1}+x_{2}\right)
\end{array}\right]^{\top}, \\
& \mathbf{g}(\mathbf{x})=\left[\begin{array}{ll}
d_{1} & 0 \\
0 & d_{1}
\end{array}\right],
\end{aligned}
$$

where $M>0$ and $c>0$ are the parameters of the switched input $M \operatorname{sign}\left(c x_{1}+x_{2}\right) ; \epsilon>0$ is the restitution uncertainty factor. The above system represents a controlled double integrator, operating under unilateral constraint $x_{1} \geq$ 0 with a restitution parameter $e \in(0,1)$ that can readily be interpreted in terms of dimensionless impacting double integrator (to numerically be studied in Section 3.4) where $x_{1}$ stands for a position deviation and $x_{2}$ is for its velocity. Both a piecewise continuous unmatched disturbance $\omega_{1} \epsilon$ $\mathbb{R}$ and a matched disturbance $\omega_{2} \in \mathbb{R}$ of the same class affect the system. It is well known that, in the unconstrained case, the control input $M \operatorname{sign}\left(c x_{1}+x_{2}\right)$ imposes disturbanceindependent sliding motions on system (9) thus specified to slide along the linear surface $c x_{1}+x_{2}=0$, provided that only matched disturbances are admitted with an upper bound on their magnitude not exceeding the control gain $M$.

The aim of this section is to demonstrate that the socalled first-order sliding mode controller $M \operatorname{sign}\left(c x_{1}+x_{2}\right)$, while driving the above system, is capable of not only rejecting matched uniformly bounded disturbances, but also attenuating restitution uncertainties and unbounded disturbances, including mismatched ones. For this purpose, the continuous-time output (6) is further specified to consist of the position deviation and the sliding variable $s=c x_{1}+x_{2}$, thus taking the form

$$
\mathbf{z}=\mathbf{h}(\mathbf{x})=\left[\begin{array}{ll}
x_{1} & s
\end{array}\right]^{T},
$$

whereas the discrete output (8) remains the same. To summarize, the underlying continuous-time system is represented as follows:

$$
\dot{\mathbf{x}}=\left[\begin{array}{c}
x_{2}+d_{1} w_{1}(t) \\
u+d_{2} w_{2}(t)
\end{array}\right],
$$

and it is driven by

$$
u=-M \operatorname{sign}(s),
$$

where the switching surface is governed by

$$
s=c x_{1}+x_{2}=0 .
$$

Once the reset surface $x_{1}=0$ is achieved, the instantaneous state transition is given by

$$
\begin{aligned}
& x_{1}\left(t^{+}\right)=x_{1}\left(t^{-}\right), \\
& x_{2}\left(t^{+}\right)=-e x_{2}\left(t^{-}\right)+\epsilon w^{d},
\end{aligned}
$$

whereas the discrete output is

$$
z_{t}^{d}=x_{2}\left(t^{+}\right)
$$

It is well known [34] that the collision-free system (47), (48) is globally asymptotically stable with the state-dependent controller gain $M=M(\mathbf{x})>c\left|x_{2}\right|+d_{2} \sup _{t \geq 0}\left|\omega_{2}(t)\right|$ whenever $d_{1}=0$ (no unmatched disturbances $\omega_{1}$ affect the system) and $c>0$. 


\subsection{Verification of Hypotheses}

3.2.1. Verification of Hypothesis (H1). Given $\gamma>\sqrt{2} \epsilon$, relation (25) is straightforwardly verified. Thus, under a fixed restitution uncertainty factor $\epsilon$, Hypothesis (H1) imposes a lower estimate on an admissible disturbance attenuation level. Since the system in question is not capable of counteracting impact dynamics instantaneously such an attenuation level cannot be made arbitrarily small and its estimate relies on the uncertainty factor at the collision instants. The smaller the uncertainty factor is during the collisions, the better the collision uncertainty is attenuated.

3.2.2. Verification of Hypothesis (H2) with the HamiltonJacobi Inequality beyond the Switching Surface. Let us first demonstrate that beyond the switching manifold (49) the positive definite function

$$
V=x_{1}^{2}+|s|
$$

satisfies the Hamilton-Jacobi inequality (22). Indeed, substituting (44)-(46), (52) into the left-hand side of (22), one has

$$
\begin{gathered}
\mathscr{H}=2 x_{1} x_{2}+c x_{2} \operatorname{sign}(s)+\left[M-\frac{1}{4 \gamma^{2}}\left(c^{2} d_{1}^{2}+d_{2}^{2}\right)\right] \\
+\frac{1}{\gamma^{2}} d_{1}^{2} x_{1}^{2}+\frac{1}{\gamma^{2}} c d_{1}^{2} x_{1} \operatorname{sign}(s)+x_{1}^{2}+S^{2},
\end{gathered}
$$

where the Hamiltonian $\mathscr{H}$ stands for the left-hand side of the Hamilton-Jacobi inequality (22). Then, taking into account the fact that $s=c x_{1}+x_{2}$ and using straightforward manipulations, involving the well-known inequality $2 x_{1} x_{2} \leq$ $x_{1}^{2}+x_{2}^{2}$, it follows that

$$
\begin{aligned}
\mathscr{H} \leq & -\left[M-\frac{1}{4 \gamma^{2}}\left(c^{2} d_{1}^{2}+d_{2}^{2}\right)\right] \\
& +R^{2}\left(4+\frac{1}{\gamma^{2}} d_{1}^{2}+2 c^{2}\right)+c R\left(1+\frac{1}{\gamma^{2}} d_{1}^{2}\right)<0 .
\end{aligned}
$$

Within the ball

$$
\mathscr{B}_{R}=\{\mathbf{x}:\|\mathbf{x}\| \leq R\}
$$

of radius $R$, inequality (54) is simplified to

$$
\mathscr{H} \leq-M+\eta_{2} R^{2}+\eta_{1} R+\eta_{0}
$$

where

$$
\begin{aligned}
& \eta_{0}=\frac{1}{4 \gamma^{2}}\left(c^{2} d_{1}^{2}+d_{2}^{2}\right), \\
& \eta_{1}=c\left(1+\frac{1}{\gamma^{2}} d_{1}^{2}\right), \\
& \eta_{2}=\left(4+\frac{1}{\gamma^{2}} d_{1}^{2}+2 c^{2}\right) .
\end{aligned}
$$

So, the Hamiltonian $\mathscr{H}$ proves to be negative definite within ball (55) provided that the controller gain $M$ is chosen according to

$$
M>\eta_{2} R^{2}+\eta_{1} R+\eta_{0}
$$

Thus, under condition (58), the Hamilton-Jacobi inequality (22) is shown to hold outside the switching surface (49), locally within the region $\mathscr{B}_{R}$.

3.2.3. Verification of Hypothesis (H2) with the HamiltonJacobi Inequality on the Switching Surface. The sliding mode equation, governing the system dynamics on the switching surface (49), is obtained by applying the equivalent control method. Thus, if confined to the switching manifold (49), the double integrator (47) reduces to the first-order system

$$
\dot{x}_{1}=-c x_{1}+d_{1} \omega_{1}
$$

and its output (46) is then specified to

$$
\mathbf{z}=\left[\begin{array}{ll}
x_{1} & 0
\end{array}\right]^{\top} .
$$

In turn, the positive definite function (52) on the sliding modes $s(x)=0$ is simplified to

$$
V=x_{1}^{2}
$$

Let us now demonstrate that the Hamilton-Jacobi inequality (24), while being specified for the sliding mode equation (59), is solved with the positive definite function (61). By substituting (61) into the Hamilton-Jacobi inequality (24), thus specified, one derives

$$
\mathscr{H}=-\left(2 c-\frac{1}{\gamma^{2}} d_{1}^{2}-1\right) x_{1}^{2}<0
$$

provided the surface parameter is chosen according to

$$
c>\frac{1}{2}\left(\frac{1}{\gamma^{2}} d_{1}^{2}+1\right) \text {. }
$$

The validity of the Hamilton-Jacobi inequality (24) is thus straightforwardly verified on the switching surface (49) subject to parameter choice (63).

3.2.4. Verification of Hypothesis (H3). For the Lyapunov function (52) and function $\boldsymbol{\mu}(\mathbf{x})$, given by (43), condition (26) of Hypothesis (H3) is specified to $\left|x_{2}\right| \geq\left|e x_{2}\right|$. Since the restitution parameter $e \in(0,1)$ the validity of this hypothesis is thus guaranteed.

3.3. $\mathscr{L}_{2}$-Gain Analysis of the Overall System. Due to derivations of Sections 3.2.1-3.2.4, Theorem 5 becomes applicable to the impacting double integrator (46)-(51). By applying Theorem 5, the following result is established.

Theorem 7. Given arbitrary $\gamma>\sqrt{2} \epsilon$ and radius $R>0$, let the sliding mode controller parameters $M$ and $c$ be chosen to ensure that inequalities (58) and (63) are satisfied. Then the 

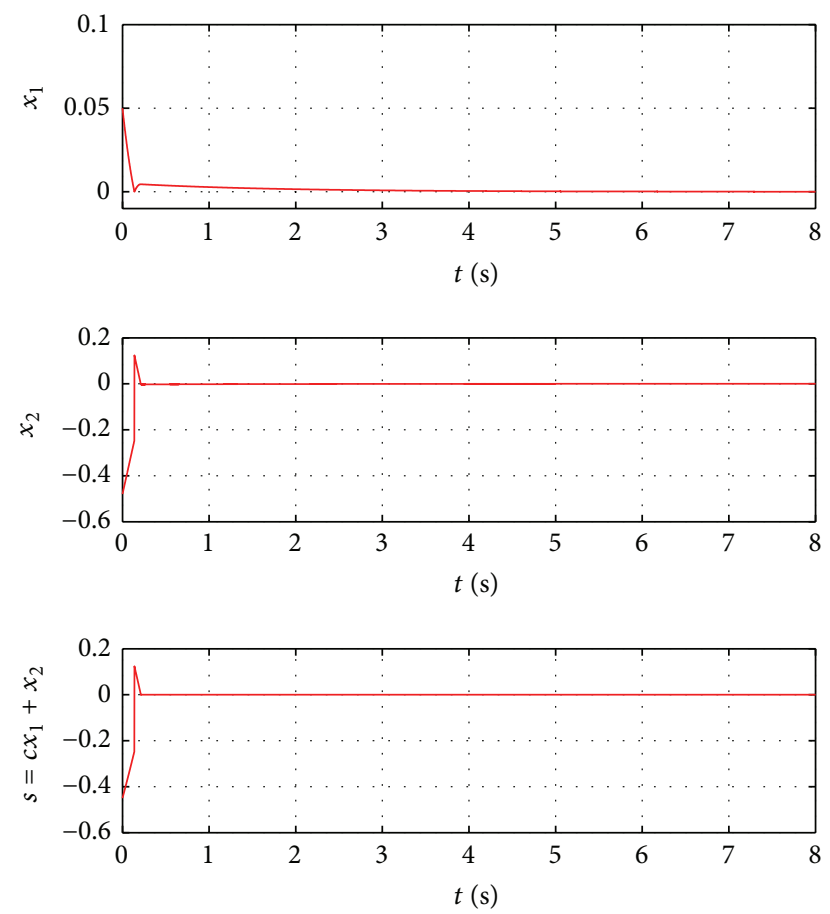

FIGURE 1: Dynamics of the undisturbed closed-loop system.

disturbance-free system (47)-(50) with $w_{1}=w_{2}=0$ and $w_{t}^{d}=$ 0 is asymptotically stable and its perturbed version possesses $\mathscr{L}_{2}$-gain less than $\gamma$ with respect to outputs (46), (51) locally within the ball $\mathscr{B}_{R}$ of radius $R$.

It is important to remark that tuning of the controller synthesis in the form (48) is greatly simplified for secondorder systems once the disturbance gains $d_{1}$ and $d_{2}$ and the region $R$ of interest are a priori fixed. This simplicity can be conserved for a system with several degrees of freedom, if there exists a prefeedback control input such that the original stabilization problem is decoupled to the stabilization of several double integrators, controlled independently. An example of such a decoupling technique applied to the finitetime stabilization of an underactuated biped robot is found in [35]; however, such a generalization is beyond the scope of the present investigation. For systems with many degrees of freedom, the verification of Hypothesis (H3) does not appear to be straightforward, and online adaptation of the reference trajectory is in order to enforce this hypothesis [36].

3.4. Numerical Performance Analysis. The performance of the hybrid system (47)-(50) is numerically tested under the parameter values $d_{1}=d_{2}=1$ in the presence of the unmatched and matched disturbances

$$
\begin{aligned}
& w_{1}=0.5 x_{2}, \\
& w_{2}=1.8 \sin (2 t), \\
& w^{d}=-0.25 x_{2} .
\end{aligned}
$$

In the simulation runs, the initial conditions were set to $x_{1}(0)=0.05$ and $x_{2}(0)=-0.48$, and the numerical study
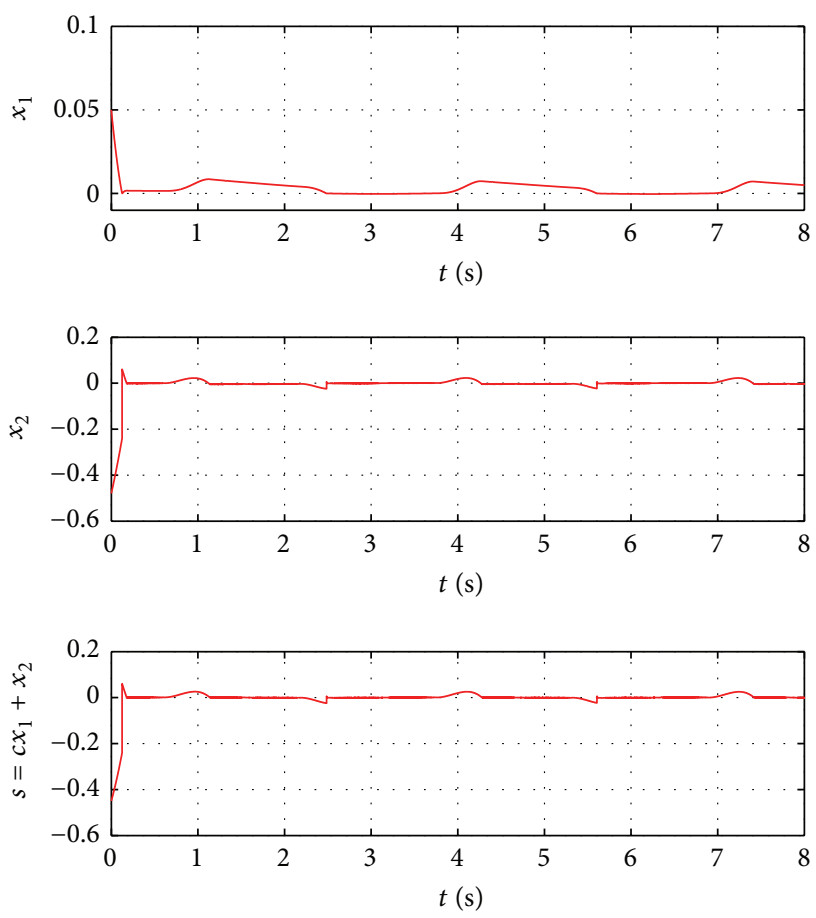

Figure 2: Dynamics of the closed-loop system affected by unmatched and matched disturbances (64).

was confined to the ball $\mathscr{B}_{R}$ of radius $R=0.5$. Specifying the desired attenuation level with $\gamma=5$, and the controller parameter $c=0.6$, thus complying with (63), the auxiliary variables (57) are found, $\eta_{0}=0.0136, \eta_{1}=0.624$, and $\eta_{2}=$ 5.24. Therefore, by setting the controller parameter $M=1.7$, all the conditions of Theorem 7 were satisfied.

Figure 1 illustrates the asymptotic stability of the disturbance-free system $\left(w_{1}=w_{2}=w^{d}=0\right)$. With the preselected initial conditions, there appears to be just one impact approximately at $t=0.12 \mathrm{~s}$, which corresponds to the vertical line in $x_{2}$ plot, and then the trajectory attains the sliding surface $s=0$ approximately at $t=0.29 \mathrm{~s}$ and stays there forever. While the sliding variable remains at its zero value, the trajectory approaches the origin asymptotically, as predicted by the theory.

The system behavior when affected by both matched and unmatched disturbances, including uncertainties in the restitution law, is illustrated in Figure 2. It is observed that the sliding motion along the surface $s=0$ is no longer in force due to the presence of the unmatched disturbances. Although the disturbed trajectory does not escape to zero anymore, the state variables remain bounded and small, so that good performance is presented despite the disturbances, also causing additional impacts. The desired disturbance attenuation is thus concluded from Figure 2.

In order to illustrate the advantages of the proposed design on the disturbance attenuation in the closed-loop system, the obtained results were compared against a controller (48), (49) with a choice of $M=1.2$ and $c=0.4$ such that inequalities (58) and (63) are not satisfied anymore. 


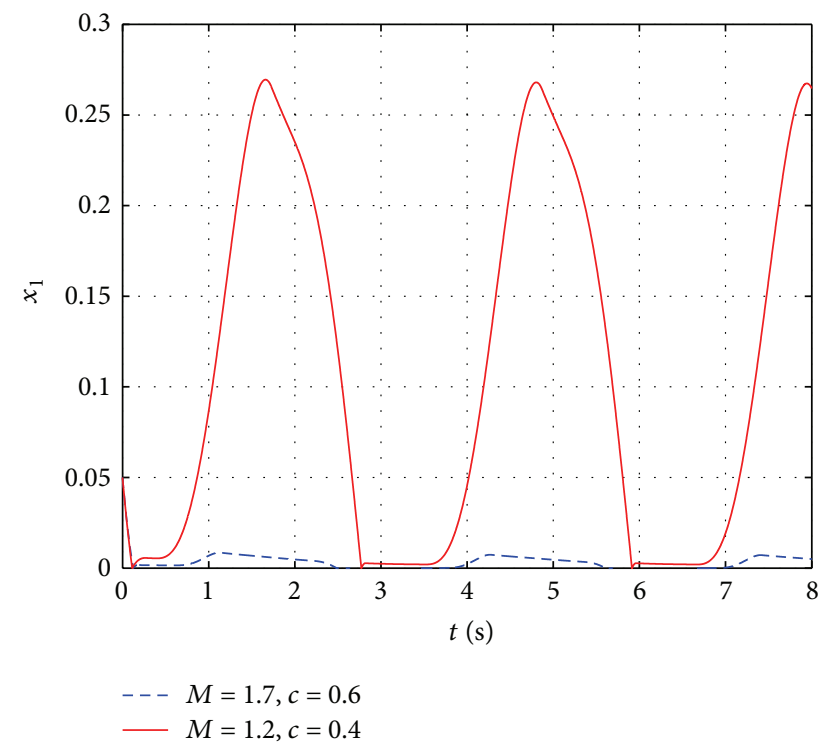

FIGURE 3: Comparison of the position errors of the closed-loop system using different values of $M$ and $c$, affected by unmatched and matched disturbances (64).

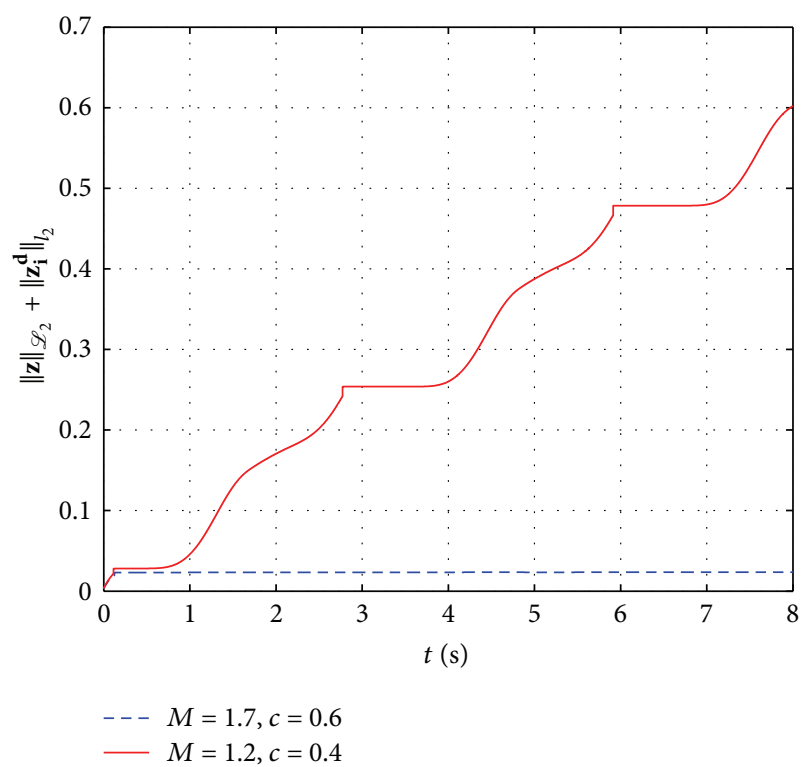

FIGURE 4: Comparison of $\mathscr{L}_{2}$ norms of the closed-loop system using different values of $M$ and $c$, affected by unmatched and matched disturbances (64).

The comparison results are presented in Figures 3 and 4, clearly showing that the performance of the closed-loop system is greatly improved once the controller parameters are properly tuned in accordance with Theorem 7.

\section{Conclusion}

In this paper, $\mathscr{L}_{2}$-gain analysis is developed for hybrid mechanical systems, admitting sliding modes and collision phenomena. Sufficient conditions for such a hybrid system to be internally asymptotically stable and to possess $\mathscr{L}_{2}$-gain less than an a priori given disturbance attenuation level are carried out beyond and on the sliding manifold. The resulting conditions are properly unified in terms of appropriate solvability of a Hamilton-Jacobi partial differential inequality. Effectiveness of the proposed analysis is illustrated in the numerical study of an impacting double integrator, driven by a sliding mode controller. It is shown that the desired disturbance attenuation level is satisfactorily achieved in the presence of external disturbances and impact uncertainties.

Bipedal robots constitute a popular benchmark of mechanical systems operating under unilateral constraints where a desired orbit to follow between impacts should be attained at a sufficiently rapid rate before the next contact occurs between the swing leg and the ground [37]. Since bipedal robots represent complex mechanical systems with many degrees of freedom, flexibility instants, and illidentified physical parameters such as inertia moments, masses, frictions, and velocity restitution coefficients (see [38]), robust control of a bipedal robot remains an active research area. A potentially interesting application of the present results to bipedal robots is among challenging problems, calling for further investigation.

\section{Conflict of Interests}

The authors declare that there is no conflict of interests regarding the publication of this paper.

\section{Acknowledgment}

The authors acknowledge the financial support of CONACYT Grant no. 165958.

\section{References}

[1] N. H. El-Farra, P. Mhaskar, and P. D. Christofides, "Output feedback control of switched nonlinear systems using multiple lyapunov functions," Systems \& Control Letters, vol. 54, no. 12, pp. 1163-1182, 2005.

[2] P. Mhaskar, N. H. El-Farra, and P. D. Christofides, "Stabilization of nonlinear systems with state and control constraints using Lyapunov-based predictive control," Systems \& Control Letters, vol. 55, no. 8, pp. 650-659, 2006.

[3] Y. Li, T. Li, and X. Jing, "Indirect adaptive fuzzy control for input and output constrained nonlinear systems using a barrier Lyapunov function," International Journal of Adaptive Control and Signal Processing, vol. 28, no. 2, pp. 184-199, 2014.

[4] B. Niu and J. Zhao, "Barrier lyapunov functions for the output tracking control of constrained nonlinear switched systems," Systems \& Control Letters, vol. 62, no. 10, pp. 963-971, 2013.

[5] B. Niu and J. Zhao, "Tracking control for output-constrained nonlinear switched systems with a barrier Lyapunov function," International Journal of Systems Science, vol. 44, no. 5, pp. 978985, 2013.

[6] Q. Su, M. Wang, G. M. Dimirovski, X. Dong, and J. Zhao, "Tracking of output-constrained switched nonlinear systems in strict-feedback form," in Proceedings of the 31st Chinese Control Conference (CCC 2012), pp. 367-372, Hefei, China, July 2012. 
[7] H. Richter, "A multi-regulator sliding mode control strategy for output-constrained systems," Automatica, vol. 47, no. 10, pp. 2251-2259, 2011.

[8] X. Zhang, J. Zhao, and G. M. Dimirovski, " $L_{2}$-gain analysis and control synthesis of uncertain switched linear systems subject to actuator saturation," International Journal of Systems Science, vol. 43, no. 4, pp. 731-740, 2012.

[9] L. Rodrigues and E.-K. Boukas, "Piecewise-linear $H_{\infty}$ controller synthesis with applications to inventory control of switched production systems," Automatica, vol. 42, no. 8, pp. 1245-1254, 2006.

[10] R. Goebel, R. G. Sanfelice, and A. R. Teel, "Hybrid dynamical systems," IEEE Control Systems Magazine, vol. 29, no. 2, pp. 2893, 2009.

[11] K. A. Hamed and J. W. Grizzle, "Robust event-based stabilization of periodic orbits for hybrid systems: application to an underactuated 3D bipedal robot," in Proceedings of the 1st American Control Conference (ACC '13), pp. 6206-6212, Washington, DC, USA, June 2013.

[12] R. Naldi and R. G. Sanfelice, "Passivity-based control for hybrid systems with applications to mechanical systems exhibiting impacts," Automatica, vol. 49, no. 5, pp. 1104-1116, 2013.

[13] D. Nešić, A. R. Teel, G. Valmorbida, and L. Zaccarian, "Finitegain stability for hybrid dynamical systems," Automatica, vol. 49, no. 8, pp. 2384-2396, 2013.

[14] T. Basar and P. Bernhard, $H_{\infty}$-Optimal Control and Related Minimax Design Problems: A Dynamic Game Approach, Modern Birkhäuser Classics, Birkhäuser, Basel, Switzerland, 1995.

[15] A. Isidori and A. Astolfi, "Disturbance attenuation and $H_{\infty}{ }^{-}$ control via measurement feedback in nonlinear systems," IEEE Transactions on Automatic Control, vol. 37, no. 9, pp. 1283-1293, 1992.

[16] A. J. Van Der Schaft, "On a state space approach to nonlinear $H_{\infty}$ control," Systems \& Control Letters, vol. 16, no. 1, pp. 1-8, 1991.

[17] E. Bernuau, D. Efimov, W. Perruquetti, and A. Polyakov, "On homogeneity and its application in sliding mode control," Journal of the Franklin Institute, vol. 351, no. 4, pp. 1866-1901, 2014.

[18] J. P. Hespanha, D. Liberzon, and A. R. Teel, "Lyapunov conditions for input-to-state stability of impulsive systems," Automatica, vol. 44, no. 11, pp. 2735-2744, 2008.

[19] F. Castaños and L. Fridman, "Dynamic switching surfaces for output sliding mode control: an $\mathrm{H}_{\infty}$ approach," Automatica, vol. 47, no. 9, pp. 1957-1961, 2011.

[20] T. Osuna and Y. Orlov, " $L_{2}$-gain analysis of sliding mode controllers," in Proceedings of the 13th International Workshop on Variable Structure Systems (VSS '14), pp. 1-6, IEEE, Nantes, France, June-July 2014.

[21] O. Montano, Y. Orlov, and Y. Aoustin, "Nonlinear output feedback $H_{\infty}$-control of mechanical systems under unilateral constraints," in Proceedings of the 1st IFAC Conference on Modelling, Identification and Control of Nonlinear Systems, pp. 284-289, Saint Petersburg, Russia, June 2015.

[22] B. Brogliato, Nonsmooth Mechanics: Models, Dynamics and Control, Springer, New York, NY, USA, 1999.

[23] Y. Orlov and L. Aguilar, Advanced $H_{\infty}$ Control-Towards Nonsmooth Theory and Applications, Birkhäuser, 2014.

[24] F. H. Clarke, Optimization and Nonsmooth Analysis, vol. 5, SIAM, 1990.
[25] Y. Orlov, Discontinuous Systems-Lyapunov Analysis and Robust Synthesis Under Uncertainty Conditions, Springer, New York, NY, USA, 2009.

[26] A. Filippov, "Differential equations with discontinuous righthand side," Matematicheskii Sbornik, vol. 93, no. 1, pp. 99-128, 1960.

[27] J. C. Willems, "Dissipative dynamical systems part I: general theory," Archive for Rational Mechanics and Analysis, vol. 45, no. 5, pp. 321-351, 1972.

[28] D. J. Hill and P. J. Moylan, "Connections between finite-gain and asymptotic stability," IEEE Transactions on Automatic Control, vol. 25, no. 5, pp. 931-936, 1980.

[29] D. Nešić, L. Zaccarian, and A. R. Teel, "Stability properties of reset systems," Automatica, vol. 44, no. 8, pp. 2019-2026, 2008.

[30] S. Yuliar, M. R. James, and J. W. Helton, "Dissipative control systems synthesis with full state feedback," Mathematics of Control, Signals and Systems, vol. 11, no. 4, pp. 335-356, 1998.

[31] W. Lin and C. I. Byrnes, " $H_{\infty}$-control of discrete-time nonlinear systems," IEEE Transactions on Automatic Control, vol. 41, no. 4, pp. 494-510, 1996.

[32] J. S. Baras and M. R. James, "Robust output feedback control for discrete-time nonlinear systems: the finite-time case," in Proceedings of the 32nd IEEE Conference on Decision and Control, pp. 51-55, IEEE, December 1993.

[33] W. M. Haddad, V. Chellaboina, and S. G. Nersesov, Impulsive and Hybrid Dynamical Systems: Stability, Dissipativity, and Control, Princeton University Press, 2006.

[34] V. Utkin, Sliding Modes in Optimization and Control Problems, Springer, New York, NY, USA, 1992.

[35] Y. Aoustin, C. Chevallereau, and Y. Orlov, "Finite time stabilization of a perturbed double integrator-part II: applications to bipedal locomotion," in Proceedings of the 49th IEEE Conference on Decision and Control (CDC '10), pp. 3554-3559, Atlanta, Ga, USA, December 2010.

[36] A. A. Grishin, A. M. Formal'sky, A. V. Lensky, and S. V. Zhitomirsky, "Dynamic walking of a vehicle with two telescopic legs controlled by two drives," The International Journal of Robotics Research, vol. 13, no. 2, pp. 137-147, 1994.

[37] B. Morris and J. W. Grizzle, "A restricted poincaré map for determining exponentially stable periodic orbits in systems with impulse effects: application to bipedal robots," in Proceedings of the 44th IEEE Conference on Decision and Control, and the European Control Conference (CDC-ECC '05), pp. 4199-4206, Seville, Spain, December 2005.

[38] O. Montano, Y. Orlov, and Y. Aoustin, "Nonlinear state feedback $H_{\infty}$-control of mechanical systems under unilateral constraints," in Proceedings of the 19th World Congress of the International Federation of Automatic Control, pp. 3833-3838, Cape Town, South Africa, August 2014. 


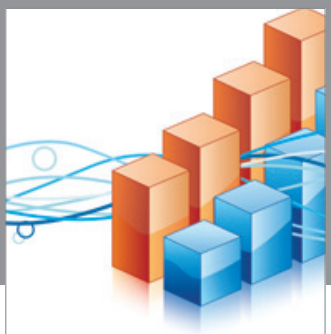

Advances in

Operations Research

vatem alat4

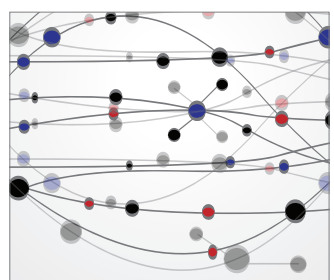

\section{The Scientific} World Journal
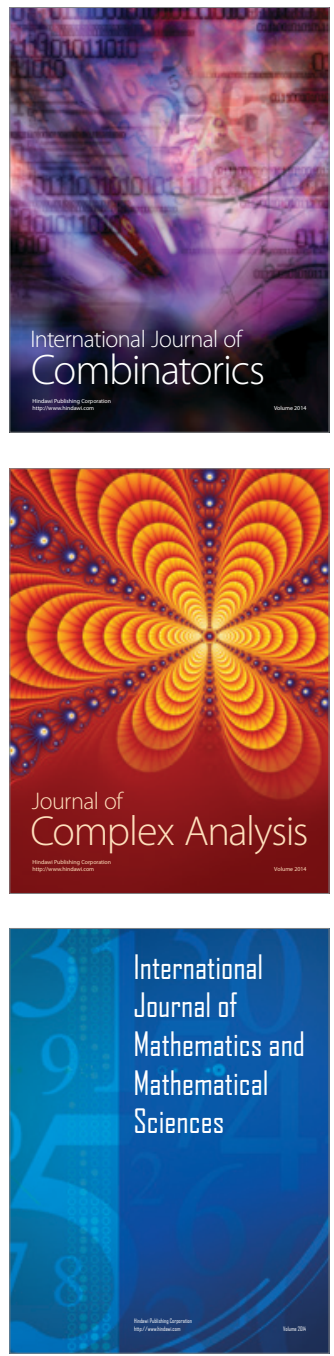
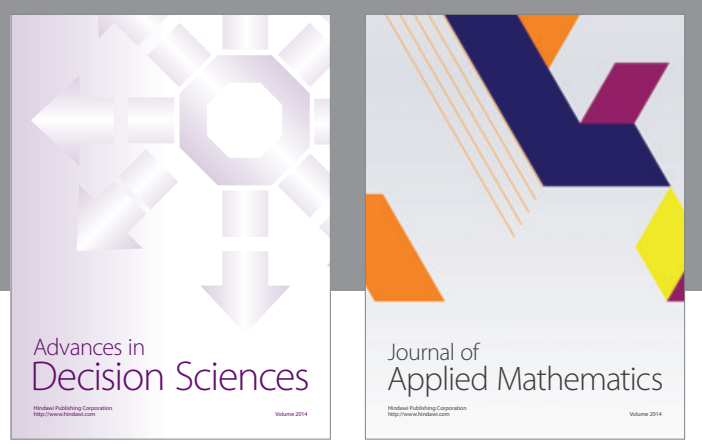

Algebra

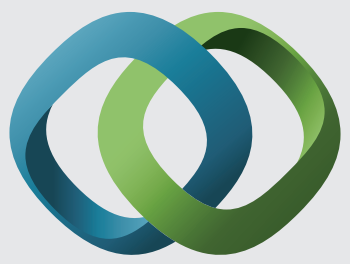

\section{Hindawi}

Submit your manuscripts at

http://www.hindawi.com
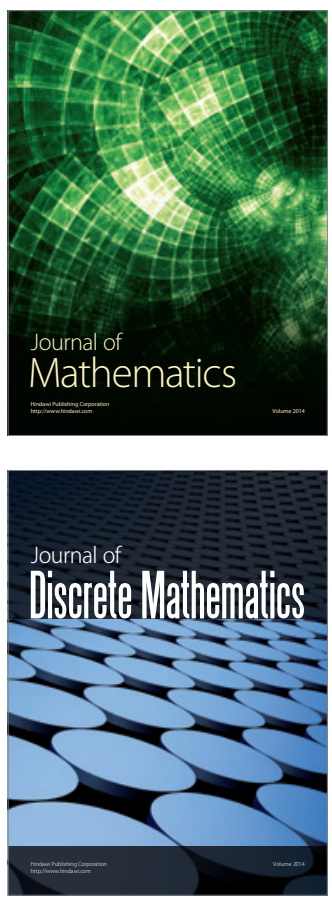

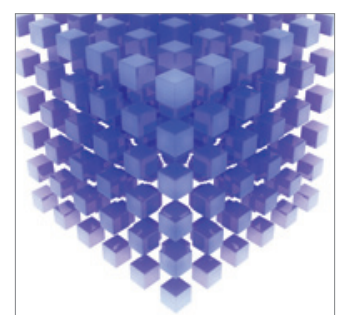

Mathematical Problems in Engineering
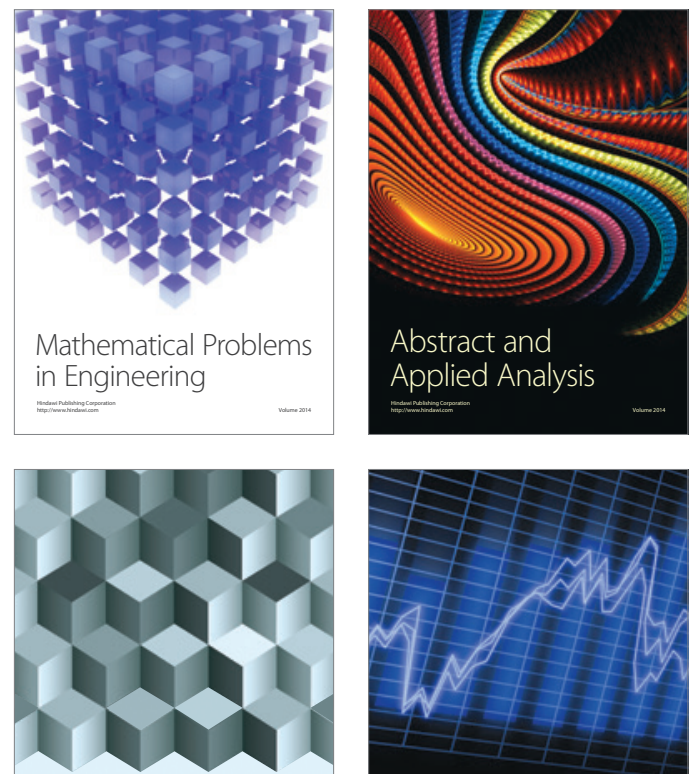

Journal of

Function Spaces

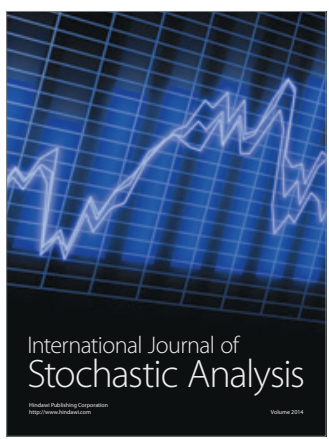

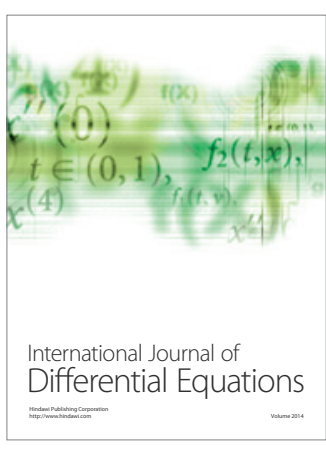
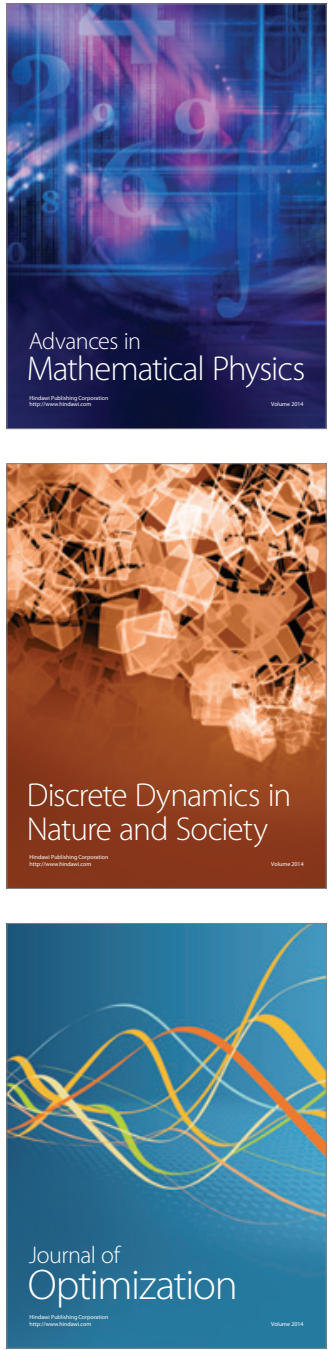